\title{
Reducing waiting lists for laparoscopic cholecystectomy: An intensive approach to aid COVID-19 recovery
}

\author{
Rachael Elizabeth Clifford (D) - Kunal Rajput · Chyu Yan Naing · Karen MacDonald · Thomas Pantak · Anil Kaul
}

Received: 17 April 2021 / Accepted: 20 April 2021 / Published online: 14 June 2021

(c) Springer-Verlag GmbH Austria, part of Springer Nature 2021

\begin{abstract}
Summary
Background Laparoscopic cholecystectomy is one of the most frequently performed operations in the United Kingdom, commonly due to symptomatic gallstones. Delay between diagnosis and definitive surgical intervention often leads to a significant readmission rate, growing financial burden and increased complexity of the ultimate surgical intervention. Resource reallocation and reduced operational capacity during the coronavirus disease 2019 (COVID-19) pandemic has led to an impending waiting list crisis. Methods In an attempt to address the backlog of cases, five intensive dedicated operating lists were allocated for laparoscopic cholecystectomies across a weekend in October 2020 at a single Trust. Prospective data were collected to include baseline demographics, operative procedure, 30-day post-operative outcomes and financial implications.

Results A total of 21 cholecystectomies were performed in total, with a majority ASA 2 (American Society of Anaesthesiologists) for predominantly biliary colic indication. All were completed laparoscopically, with a $90.5 \%$ rate for complete resection. There were no reported on-table complications and $81.0 \%$ of patients discharged as a day case. Thirty day follow-up revealed a complication rate of $9.5 \%$, with 2 patients requiring oral antibiotics for a superficial wound infection. The 30 day COVID-19 rate was $14.3 \%$. Compared to completion on an average weekday list, the total weekend was estimated to have saved over $£ 70,000$ in overall costs.
\end{abstract}

\footnotetext{
R. E. Clifford $(\bowtie) \cdot$ K. Rajput · C. Y. Naing $\cdot$ K. MacDonald T. Pantak · A. Kaul

St Helens and Knowsley Teaching Hospitals NHS Trust, Warrington Road, Prescot, L35 5DR, UK

rachael.clifford@nhs.uk
}

Conclusion Our study showed that weekend focused operating, with a caveat of careful patient selection and high-quality multidisciplinary working, can be a feasible solution to long waiting lists due to COVID19 pandemic. It was safe, with avoidance of increased burden on emergency resources, and significantly increased theatre efficiency.

Keywords Cholecystectomy · Gallstones . Coronavirus disease 2019 • Waiting lists · Training

\section{Introduction}

A laparoscopic cholecystectomy is one of the most commonly performed operations in the United Kingdom (UK), with over 50,000 performed annually [1]. Gallstone disease presents a significant burden on the National Health Service (NHS) and there is often a significant delay between patients presenting with a gallstone complication, being added to a surgical waiting list and undergoing their operation. Almost 10\% [2] of those patients will represent during their time on that waiting list with further complications requiring hospital admission, adding to the cost of their treatment journey and also increasing the complexity of their ultimate surgical intervention [3, 4]. Previous analysis of the impact of delay to elective surgery for patients with gallstones was also found to be associated with significant psychological and social consequences [5].

The first wave of coronavirus disease 2019 (COVID19) and national Governmental lockdown in March 2020 resulted in an unparalleled situation due to an initial paucity of underlying knowledge regarding the specific risks of elective and laparoscopic surgery. With reduced surgical list capacity, critical care availability and staff reallocation, even cancer service provision was severely affected and halted in many trusts. Patients were wary to attend healthcare 
settings [6] and often opted to manage conditions conservatively to avoid hospital admission. As intensive work is done to aid recovery and prioritise cancer work, elective benign surgery continues to struggle to get back to full speed even a year down the line. In November 2019 there were 4.5 million patients waiting for elective treatment on the NHS, with an estimate of an additional 1.2 million every 3 months of the pandemic being added to that [7]. With the impending waiting list crisis and continued burden on the healthcare service due to COVID-19 there has been much debate as to the best strategy to tackle elective surgery moving forward in both an effective and timely manner.

Our study aims to analyse the role of intensive dedicated operating lists to reduce cholecystectomy waiting lists in a single Trust as a mode of COVID-19 recovery.

\section{Methods}

The first 105 patients on the waiting list for a laparoscopic cholecystectomy at our Trust were screened for suitability for elective day case surgery at a single site. At the time of screening 223 patients were on the waiting list in total. The 65 identified as potentially appropriate were then discussed at a multidisciplinary meeting with Upper Gastrointestinal (UGI) Consultant Surgeons, advanced specialist nurse practitioner and admissions department representatives to screen medical comorbidities, previous surgical history and ASA (American Society of Anaesthesiologists) grading. Twenty three patients were then listed for day-case weekend laparoscopic cholecystectomy on 17-18 October 2020.

Patients were requested to self-isolate for 14 days prior to admission, with COVID-19 PCR (Polymerase chain reaction) swabs taken 3 days before the date of surgery to confirm a negative status. Five Consultant Surgeons lead the lists each day, assisted by either a trainee registrar, staff grade registrar or advanced specialist nurse practitioner.

Prospective data collection was performed for all patients undergoing planned surgery on the weekend of the 17-18 October 2020. Each patient was followed up at 30 days with a telephone consultation, and the

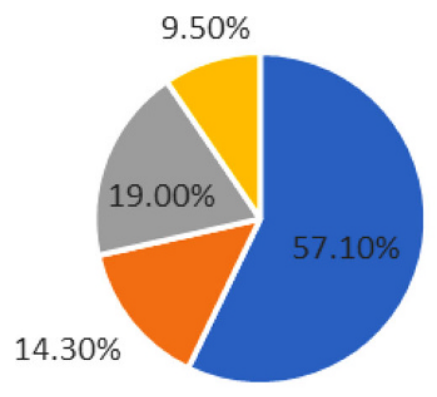

- Biliary colic

- Choledocholithiasis

- Cholecystitis

- Pancreatitis

Fig. 1 Summary of primary indication for performing cholecystectomy local medical records screened in addition to identify readmission or return to theatre.

Financial costing was obtained from the finance department for the weekend and compared to a standard elective laparoscopic cholecystectomy day case list on a standard working day.

\section{Results}

Of the 23 patients listed for surgery, 2 cancelled prior to their period of isolation due to concerns over COVID-19 alone, with 1 cancelling during that period for the same rationale. The 3 theatre list slots were filled with 2 elective inguinal hernias with a full period of isolation, and 1 'hot' emergency in patient cholecystectomy.

\section{Baseline demographics}

In total, 21 laparoscopic cholecystectomies were performed at St. Helens and Knowsley Trust during the weekend of 17 th and 18th October. The median age was 53 years (range 47-65), with a higher predisposition to the female gender $(85.7 \%)$. The average body mass index (BMI) for patients was $32.9 \mathrm{~kg} / \mathrm{m}^{2}$, with most patients falling in the 'class 1 obese' category. The patient selection is reflected in the majority of patients being American Society of Anaesthesiology (ASA) $1(9.5 \%)$ or $2(57.1 \%)$, with no patient ASA 4 or above.

Three patients had a history of previous venous thromboembolic (VTE) events, with 3 on anticoagulation for pulmonary embolus or atrial fibrillation requiring pre-operative management. Ten had undergone previous abdominal surgery, most commonly a caesarean section, with only 1 upper abdominal procedure in the form of a laparoscopic gastric bypass to predict potential adhesions.

\section{Preoperative assessment}

Recurrent biliary colic was the primary diagnosis and indication for cholecystectomy in the majority (57.1\%) of patients. The proportion of each biliary complication is demonstrated in Fig. 1.

Previous diagnostic imaging was performed in the form of a biliary ultrasound scan in $66.7 \%$ of patients, a median of 156.0 days prior to surgery (range 24-426 days), and magnetic resonance cholangiopancreatogram (MRCP) in $61.9 \%$ of patients, a median of 144 days prior to surgery (range 15-905 days). Pre-operative ERCP and sphincterotomy was conducted in $14.3 \%$ of patients for management of ductal stones, all within the previous 28 days. A summary of previous diagnostic resource utility is summarised in Fig. 2.

In all, $81.0 \%$ of patients had normal liver function tests 2-4 weeks pre-operatively, with 1 significantly improving post-ERCP and one only an isolated borderline rise in ALT. All patients received intravenous 
Fig. 2 Number of patients undergoing pre-operative investigations or procedures. US ultrasound, $M R C P$ magnetic resonance chonlangiopancreatogram, ERCP endoscopic retrograde cholangiopancreatography

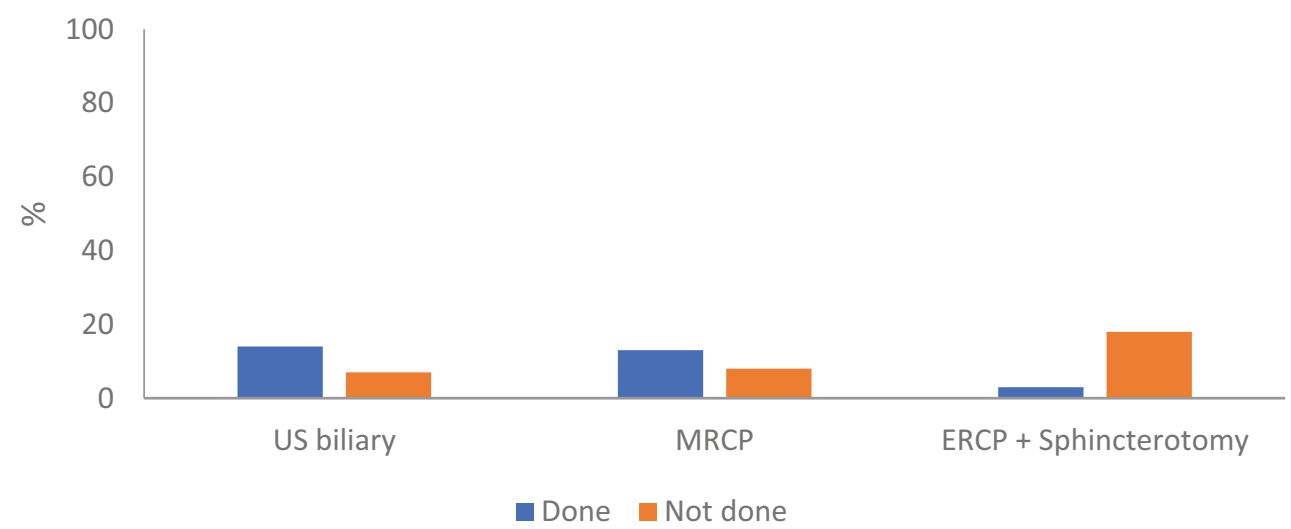

antibiotics at induction according to Trust guidelines with VTE prophylaxis.

\section{Intra-operative procedure}

All cases were performed laparoscopically via a 4 port standard approach, with no conversions to open. Nineteen cases successfully proceeded to cholecystectomy, with 2 abandoned due to inflammation and adhesions, one of which was the additional 'hot' case added from an inpatient. There were no on-table complications reported.

Median operating time was $1 \mathrm{~h} 34 \mathrm{~min}$ (range $1 \mathrm{~h} 09$ $2 \mathrm{~h} 09$ ) on the Saturday, and $1 \mathrm{~h} 30 \mathrm{~min}$ (range $0 \mathrm{~h} 50$ $2 \mathrm{~h} 06$ ) on the Sunday, with total median anaesthetic and surgical time $2 \mathrm{~h} 38$ (range $2 \mathrm{~h} 19-3 \mathrm{~h} 07$ ) and $2 \mathrm{hr} 47$ (range $1 \mathrm{~h} 45-3 \mathrm{~h} 25$ ) respectively. There was a median wait of $23 \mathrm{~min}$ between patients leaving theatre and the next entering the anaesthetic room across the whole weekend.

\section{Post-operative outcomes}

Laparoscopic cholecystectomy was performed as a day case in $81.0 \%$ of cases, with only the emergency abandoned case remaining in hospital for more than 2 further nights until day 13. Standard day case rates at the Trust for 2019/2020 for laparoscopic cholecystectomies was 53\% overall. All patients returned with benign histology.

At 30 day telephone follow-up 2 cases reported post-operative Clavien-Dindo II complications of a superficial umbilical port site infection requiring oral antibiotics (9.5\%). Two patients re-presented to A\&E with one re-admission; 1 with right-sided abdominal pain requiring escalated analgesia who was discharged directly, and 1 for investigation of pain to exclude ductal stones. Neither required further procedural intervention.

Patients were not routinely swabbed for COVID-19 on discharge. There were 2 reported cases of COVID19 in the 30 day follow-up period, both positive on day 5 , and a further at day 25 with alternative exposure risk. All patients had mild symptoms and did not go
Table 1 Summary of post-operative surgical outcomes

\begin{tabular}{|l|l|}
\hline Outcome & $\begin{array}{l}\text { Cohort } n(\%) \\
n=21\end{array}$ \\
\hline LOS & $17(81.0)$ \\
\hline 0 & $2(9.5)$ \\
1 & $1(4.8)$ \\
\hline 2 & $1(4.8)$ \\
\hline$>2$ & $2(9.5)$ \\
\hline 30 day complication rate & $1(4.8)$ \\
\hline 30 day readmission rate & $3(14.3)$ \\
\hline 30 day COVID-19 rate & LOS length of stay, COVID-19 coronavirus disease 2019
\end{tabular}

on to require hospital admission. A summary of postoperative outcomes is displayed in Table 1.

\section{Financial implications}

The overall Trust expenditure for the weekend was $30,277.41$ and overall generated income 59,589.18, resulting in a net profit of 29,311.77.

After looking at a sample of 10 previously performed laparoscopic cholecystectomies on a standard elective list and producing an average operative and total surgical time we estimated the cost of a laparoscopic cholecystectomy performed on a week day list at our Trust to be 5209. This cost included the cost of theatre use from anaesthetic start time to entry into theatre recovery, staffing and non-pay costs. Comparatively, this would result in a total expenditure of 109,389 for 21 laparoscopic cholecystectomies performed during standard working hours.

Additional administrative time for screening of patients and a MDT meeting involving 3 Consultant Surgeons to clarify patient appropriateness for listing was estimated at 1311.32; still demonstrating a financial gain to the Trust.

\section{Discussion}

During the initial wave of COVID-19 an estimated 2.3 million cancer operations were postponed worldwide due to unacceptable perioperative COVID-19 
morbidity and mortality, and reallocation of resources [8]. As streamlined COVID-19 'cold' pathways were established and alternate sites employed elective operating was able to return with favourable outcomes [8]. With repeated waves of the pandemic benign operating however has taken longer to return to full capacity, and in many centres remains at a reduced level. Recovery for benign elective services following the third COVID-19 lockdown requires a clear plan to enable effective targeting of the ever-growing backlog in a timely, yet safe manner.

Our study has demonstrated that by careful patient selection and clinician buy in, weekend focused operating does provide a viable solution for rapid waiting list reduction at a single Trust. $81.0 \%$ of patients were able to be completed as a day case procedure, with a less than $5.0 \%$ readmission rate; minimising further burden on emergency care provision. The 2 patients diagnosed with superficial wound infections, giving an all cause 30 day complication rate of just under $10 \%$, were diagnosed by their general practitioners and did not require attendance at hospital for drainage. A 30 day COVID-19 rate of $14.3 \%$ however does raise questions about the potential choice of site. All of these cases were carried out in a hospital caring for patients with COVID-19, and although strict precautions were taken, this is a significant post-operative rate. The use of a dedicated COVID-19 'cold' day case site, which is available, may act to reduce these levels.

The use of weekend operating, and additional staffing costs will impact an additional pressure on the Trust budget. The balance of increased theatre efficiency and avoidance of repeated hospital patient admissions with associated diagnostic and therapeutic interventions may however produce a positive balance overall. The key to success with this approach, as with any to target waiting lists, is careful patient selection and high quality multidisciplinary working.

The COVID-19 period has also had a substantial impact upon surgical training across all grades, with complete loss of training in elective operating during the first lockdown reported in almost $50 \%$ of cases, and over $40 \%$ of junior trainees being redeployed to alternative specialties $[9,10]$. An intensive period of repetitive training, even with segmental operative learning, for example umbilical port placement, may provide an excellent opportunity for trainees to regain their basic skills and confidence quickly after a prolonged period of reduced training opportunities.

In summary we conclude that focused weekend operating would provide a safe effective option for re- duction of waiting lists, albeit with the caveat of careful patient selection and clinician buy in.

\section{Key points}

- In an era of mounting waiting list pressures, this approach may provide merit to rapidly and effectively reduce waiting lists for a carefully selected cohort of patients in the right setting

Conflict of interest R. E. Clifford, K. Rajput, C. L. Yan Naing, K. MacDonald, T. Pantak and A. Kaul declare that they have no competing interests.

\section{References}

1. Hobbs MS, Mai Q, Knuiman MW, Fletcher DR, Ridout SC. Surgeon experience and trends in intraoperative complications in laparoscopic cholecystectomy. Br J Surg. 2006; https://doi.org/10.1002/bjs.5333.

2. Rossi BWP, BassettE, Martin M, Andrews S, Wajed S. Prompt laparoscopic cholecystectomywould reducemorbidityand save hospital resources. Ann R Coll Surg Engl.2014; https: / / doi.org/10.1308/003588414X13814021680111.

3. Gurusamy KS, Koti R, Fusai G, Davidson BR. Early versus delayed laparoscopic cholecystectomy for uncomplicated biliary colic. Cochrane Database SystRev. 2013; https://doi. org/10.1002/14651858.CD007196.pub3.

4. Somasekar K, Shankar PJ, Foster ME, Lewis MH. Costs of waiting for gall bladder surgery. Postgrad Med J. 2002; https://doi.org/10.1136/pmj.78.925.668.

5. Oudhoff JP, Timmermans DRM, Bijnen AB, Van Der Wal G. Waitingfor electivegeneral surgery: physical, psychological and social consequences. ANZ J Surg. 2004; https://doi. org/10.1111/j.1445-1433.2004.02998.x.

6. Sud A, Torr B, Jones ME, et al. Effect of delays in the 2-week-wait cancer referral pathway during the COVID19 pandemic on cancer survival in the UK: a modelling study. lancet Oncol. 2020; https://doi.org/10.1016/S14702045(20)30392-2.

7. Macdonald N, Clements C, Sobti A, Rossiter D, Unnithan A, Bosanquet N. Tackling the elective case backlog generated by Covid-19: the scale of the problem and solutions. JPublic Health. 2020; https://doi.org/10.1093/pubmed/fdaa155.

8. Glasbey JC, Nepogodiev D, Simoes JFF, et al. Elective cancersurgeryinCOVID-19-freesurgical pathwaysduring the SARS-cov-2 pandemic: an international, multicenter, comparative cohort study. J Clin Oncol. 2021; https://doi. org/10.1200/JCO.20.01933.

9. COVID-STAR Collaborative Study Group. COVID-19 impact on surgical training and recovery planning (COVIDSTAR) - A cross-sectional observational study. Int J Surg. 2021;88:105903.

10. Khan KS, Keay R, McLellan M, Mahmud S. Impact of the COVID-19 pandemic on core surgical training. Scott Med J. 2020; https://doi.org/10.1177/0036933020949217.

Publisher's Note Springer Nature remains neutral with regard to jurisdictional claims in published maps and institutional affiliations. 